\title{
GCN4-Based Expression System (pGES): Translationally Regulated Yeast Expression Vectors
}

BioTechniques 28:552-560(March 2000)

\author{
Avishai Mimran, Irit Marbach \\ and David Engelberg \\ The Hebrew University of \\ Jerusalem, Jerusalem, Israel
}

\section{ABSTRACT}

The expression of foreign proteins in Saccharomyces cerevisiae is a powerful tool for basic research and the biotechnological industry. In spite of the potential of S. cerevisiae, only a few useful expression vectors have been developed for this yeast. These vectors are based on an increasing transcription rate in combination with an increase in gene dosage. Most vectors are maintained as plasmids, which forces growth of cultures on poor selective media. Expression of the yeast Gcn4 protein is regulated at the translational level and increases strongly under amino acid starvation. Because under these conditions protein synthesis in general ceases, it is conceivable that regulatory elements that control Gcn4 expression could support selective expression of foreign genes. We cloned DNA fragments residing upstream from the GCN4 coding sequence (including the $5^{\prime}$ UTR) and ligated them to a cDNA that encodes the human serum albumin (HSA) gene. These GCN4 regulatory elements induced efficient HSA expression at the translational level under amino acid starvation. The GCN4/HSA cassette promoted efficient, inducible expression on either a multicopy or integrative plasmid. The integrated cassette induced a high level of HSA in dense cultures grown on rich media. Thus, the GCN4-based expression system ( $p G E S)$ provides high protein quantities. pGES is the first expression vector to be induced at the translational level.

\section{INTRODUCTION}

The expression of proteins in microorganisms is a powerful tool for basic research and for various medical and industrial needs. A variety of advanced expression systems have been developed in prokaryotes, in particular in the bacterium Escherichia coli $(9,20,28)$. In this organism, a foreign protein can be expressed efficiently, reaching a level of $10 \%-30 \%$ of its total protein content $(7,9,20,28)$. However, many eukaryotic proteins expressed in bacteria are not active. For various reasons, proteins expressed in bacteria must be extensively purified and assayed in rigorous and expensive quality-control systems. Therefore, although powerful, the bacterial systems are disadvantageous in many cases $(3,27)$.

An attractive alternative is the baker's yeast, Saccharomyces cerevisiae. Most foreign proteins expressed in this eukaryote are accurately processed posttranslationally and are biologically active $(3,10,21,27)$. $S$. cerevisiae has been widely used in the food industry and is regarded as a safe organism; the risk of side effects and the odds of the presence of pathogenic agents are minimal $(3,10,27)$. Although $S$. cerevisiae was used successfully for the production of several proteins for therapeutic and diagnostic uses (10), and in spite of its great potential as a protein factory, this organism is not widely used for the production of proteins. The major reason for this seems to be that the level of expression obtained in yeast is significantly lower than that obtained in bacteria $(3,10,21,23,24)$.

Only a few yeast expression vectors are available. All vectors (including bacterial and mammalian vectors) contain strong promoters (usually a promoter of a glycolitic gene; e.g., GPD1, $A D H 1$ or $P G K 1)(21,23,24)$. In addition, they contain the $2 \mu \mathrm{m}$ origin of replication or a multicopy integration system that promotes maintenance of the vector in 60-100 copies per cell $(10,22)$. Some of the vectors contain an inducible promoter, the most widely used being the GAL1-10 promoter $(21,23)$. The strategy of all yeast expression vectors is therefore based on a high transcription rate and the presence of many copies per cell.

Most yeast expression vectors are present in the cell as nonintegrated plasmids. This forces the growth of expressing cultures on synthetic media under selective conditions. When grown in laboratory flasks, these cultures enter the stationary phase at a low cell density, and the quantities of proteins obtained are low. When grown in industrial fermentors, high cell concentrations could be obtained but would require special treatments and long, expensive incubation time.

Here, we describe the development and use of a novel type of expression vector that is fully inducible and can be used in rich media. This vector is based on the regulatory elements of the GCN4 gene. GCN4 encodes a transcriptional activator with target genes that encode amino acids and nucleotides biosynthetic enzymes $(15,16)$. Under optimal growth conditions on media supplemented with amino acids and pyrimidine and purine bases, Gcn4 is not expressed. When cells are starved for amino acids, Gcn4 expression is dramatically induced, and consequently, transcription of biosynthetic genes 
commences. The expression of Gcn 4 is mainly regulated at the translational level. The mRNA levels of GCN4 are high under most growth conditions, but the translation rate of this mRNA is extremely low (17). The low translation rate is maintained by four short open reading frames (ORFs) located at the $5^{\prime}$ noncoding leader of the message (upstream ORFs 1-4; 4uORF) (4,15-17) (Figure 1). Ribosomes recognize uORF1 and begin translation, yet they dissociate from the mRNA at the stop codon and reinitiate translation at another short ORF. In most cases, ribosomes do not reach the fifth ORF, which is the GCN4 coding sequence (17). The inhibition of GCN4 translation depends on the high rate of reinitiation. Under amino acid starvation, the rate of reinitiation is reduced to a minimum, and consequently, translation activity in the cell pauses, which is an obvious protective response. Reduction in reinitiation activity is mediated by the Gcn2 kinase that phosphorylates, and thereby inhibits, the activity of initiation factor $2 \alpha$ (eIF2 $\alpha)(4,17)$. Although translation in the cell is generally ceased under these conditions, translation of GCN4 mRNA is specifically induced (15-17). This activity seems a logical response because the Gcn 4 protein is required for the induction of amino acid biosynthesis. Following the translation of Gcn 4 and the synthesis of amino acids, general translation activity restarts. In the laboratory, amino acid starvation could be easily mimicked by the treatment of yeast cells with inhibitors of biosynthetic enzymes. The most widely used compound is 3amino-1,2,4-triazole (3-AT), a competitive inhibitor of His3. Cells combat the competitive inhibition effect simply by

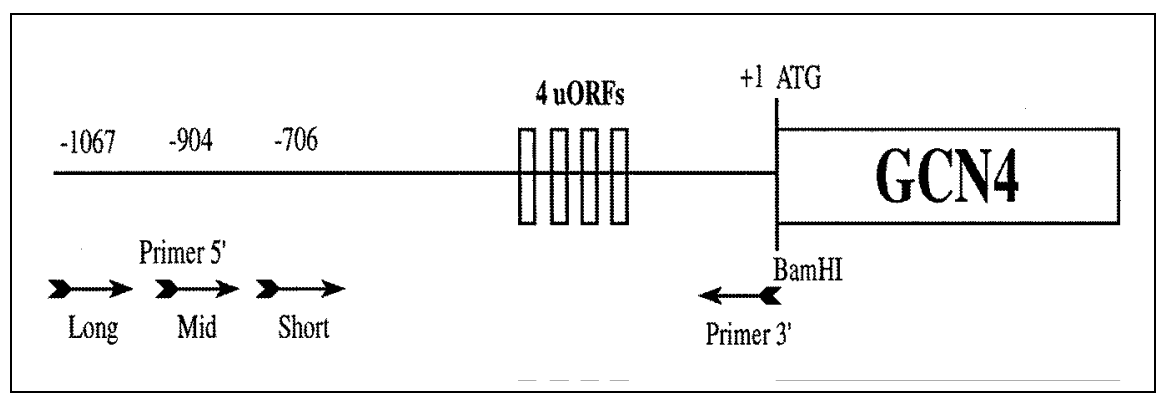

Figure 1. Schematic description of the $G C N 4$ gene and the primers used for promoter cloning. ORFs are shown as boxes and primers as arrows.

producing more His3. The GCN4 translation rate, which leads to HIS3 transcription, dramatically increases in response to 3-AT treatment $(12,15)$.

We proposed that the unusual pattern of Gen4 expression could be advantageous for the expression of a foreign protein. We reasoned that if the mRNA of a given foreign protein is selectively translated when translation of most cellular mRNAs is halted, its concentration should be high. To test this idea, we have cloned the regulatory elements of GCN4 (from - 1067 to $-1 ;+1$ is the initiation of translation) and ligated this fragment to the coding sequence of the human serum albumin (HSA) gene. We show that under the control of $G C N 4$ regulatory elements, expression of HSA is rapidly and efficiently induced by 3-AT. We further show that induction occurs at the translational level and could be finely controlled by using various $3-\mathrm{AT}$ concentrations. Importantly, this vector induces efficient expression as an integrated copy, which allows growth on rich media to high cell density.

\section{MATERIALS AND METHODS}

\section{Yeast Strains and Growth Conditions}

The SP1 strain (MATa, his3, leu2, ura3, trp1, ade8, can $^{r}$; obtained from M. Wigler) (18) was used as a source of genomic DNA from which the GCN4 upstream sequences were cloned. Strain BJ2168 (MATa, prc1-407, prb1-1122, pep4-3, leu2, trp1, ura3-52; obtained from the yeast genetic stock center at the University of California-Berkeley) (29) was used as a host for the expres-

Vol. 28, No. 3 (2000) sion plasmids. Yeast cultures were grown on either $1 \%$ yeast extract, $2 \%$ bactopeptone, $2 \%$ glucose (YPD medium) or $0.17 \%$ yeast nitrogen base without amino acids and ammonium sulfate (Difco, Detroit, MI, USA), $0.5 \%$ $\mathrm{NH}_{4}\left(\mathrm{SO}_{4}\right)_{2}, 2 \%$ glucose and the required amino acids and uracil $(40 \mathrm{mg} / \mathrm{L})$ (SD medium). Strains that harbor plasmids were grown on SD(-uracil). For expression experiments, cultures were harvested at the logarithmic phase (unless mentioned otherwise) and resuspended in SD(-uracil, -histidine) [or in $\mathrm{SD}$ (-histidine) for integrated vectors], supplemented with 3-AT (see details of each experiment).

\section{PCR Cloning of GCN4 Fragments and Plasmid Constructions}

PCRs were performed using genomic DNA of the yeast strain SP1 as a template and the following primers. Primer 3' (5'-GCGCCGGATCCTTTATTTGTATTTAATTTATTTTCTTGAGC-3') contains a BamHI site and was used in all PCRs (Figure 1). The long promoter (5'-GCGCCGACTAGTTTGCCACGTACATGACATTA-3') was used to amplify the sequence from -1067. The middle-sized promoter $\left(5^{\prime}\right.$ GCGCCGACTAGTGCCGAACACCACCGGCATCTTG-3') was used to amplify the sequence from -904 . The short promoter (5'-GCGCCGACTAGTCGGAAGATAAATACTCCAAC-3') was used to amplify the sequence from -706. These three primers contain SpeI sites. They were used at the $5^{\prime}$ side of the GCN4 promoter (Figure 1). PCR products were digested with SpeI and BamHI and ligated to $\mathrm{pBluescript}^{\circledR}$ (Stratagene, La Jolla, CA, USA) to obtain pBSG-Short (pBSG-S), pBSGMiddle (pBSG-M) and pBSG-Long (pBSG-L). The cloned DNAs were sequenced and found to be identical to the sequence of this region as it appears in the Saccharomyces genome database.

To ligate the coding sequence of $H S A$ downstream from the GCN4 sequences, the cDNA of $H S A$ was cloned as a BamHI-EcoRI fragment to pBSGS, pBSG-M and pBSG-L. These chimeric genes contained about $70 \mathrm{bp}$ of $5^{\prime}$ UTR sequences of $H S A$, which were found to reduce the expression level (data not shown). To remove this 
sequence from each of the three plasmids, a 885 bp BamHI-NcoI fragment that contains the entire $5^{\prime}$ UTR and 815 bp of the HSA coding sequence was replaced with a $815 \mathrm{bp}$ PCR product that contains only the first $815 \mathrm{bp}$ of the $H S A$ coding sequence. Primers used for this PCR were HSA-ATG (5'-GCGCGCGGATCCATGAAGTGGGTAACCTTTATTTCCC-3') and HSA-815 (5'-GCAGATCTCCATGGCAGCATTCC-3').

The plasmids obtained were designated pBSG-S-HSA, pBSG-M-HSA and pBSG-L-HSA. The GCN4-HSA moiety of each of these plasmids was removed as a SpeI-EcoRI fragment and inserted into pRS316 (a centromeric yeast plasmid) (25), pRS306 (an integrated yeast vector) (25) and pRS426 (a $2 \mu \mathrm{m}$-based vector) (2). The terminator of $A D H I$ ( $0.6 \mathrm{~kb} E c o$ RI fragment) was ligated to the EcoRI site of each of the nine plasmids obtained. The final constructs were designated as follows: $(i)$ pGES306-S-HSA (for GCN4-based expression system, derived from pRS306, which contains the short GCN4 promoter and the HSA cDNA; (ii) pGES306-M-HSA, which is similar to pGES306-S-HSA but contains the medium-sized GCN4 promoter); (iii) pGES306-L-HSA, which contains the long GCN4 promoter; (iv) pGES316-SHSA; (v) pGES316-M-HSA; (vi) pGES316-L-HSA; (vii) pGES426-SHSA; (viii) pGES426-M-HSA; and (ix) pGES426-L-HSA. Figure 2 depicts pGES306-L-HSA, pGES316-L-HSA and pGES426-L-HSA.

Integrated vectors were digested with StuI before yeast transformation. This digestion directs the vector to the $U R A 3$ locus of the genome. To express $H S A$ under the $A D H 1$ promoter, a BamHI-EcoRI fragment containing the HSA cDNA was rendered blunt by DNA PolI (Klenow fragment) and inserted into the $S m a \mathrm{I}$ site of pAD4 $\Delta$ (1).

\section{Western Blot Analysis}

Protein extracts were prepared as previously described (8). From each extract, $30 \mu \mathrm{g}$ were used for Western blot analysis. The SDS-PAGE gels contained $10 \%$ acrylamide. Protein blotting was performed with a semi-dry blotter (LKB, Sweden) for $2 \mathrm{~h}$ under constant current $(\mathrm{mA}=0.8 \times$ gel area in $\mathrm{cm}^{2}$ ). Polyclonal anti-HSA antibodies (RAHu/albumin) were purchased from Nordic Immunology (Tilburg, The Netherlands) and used in a dilution of

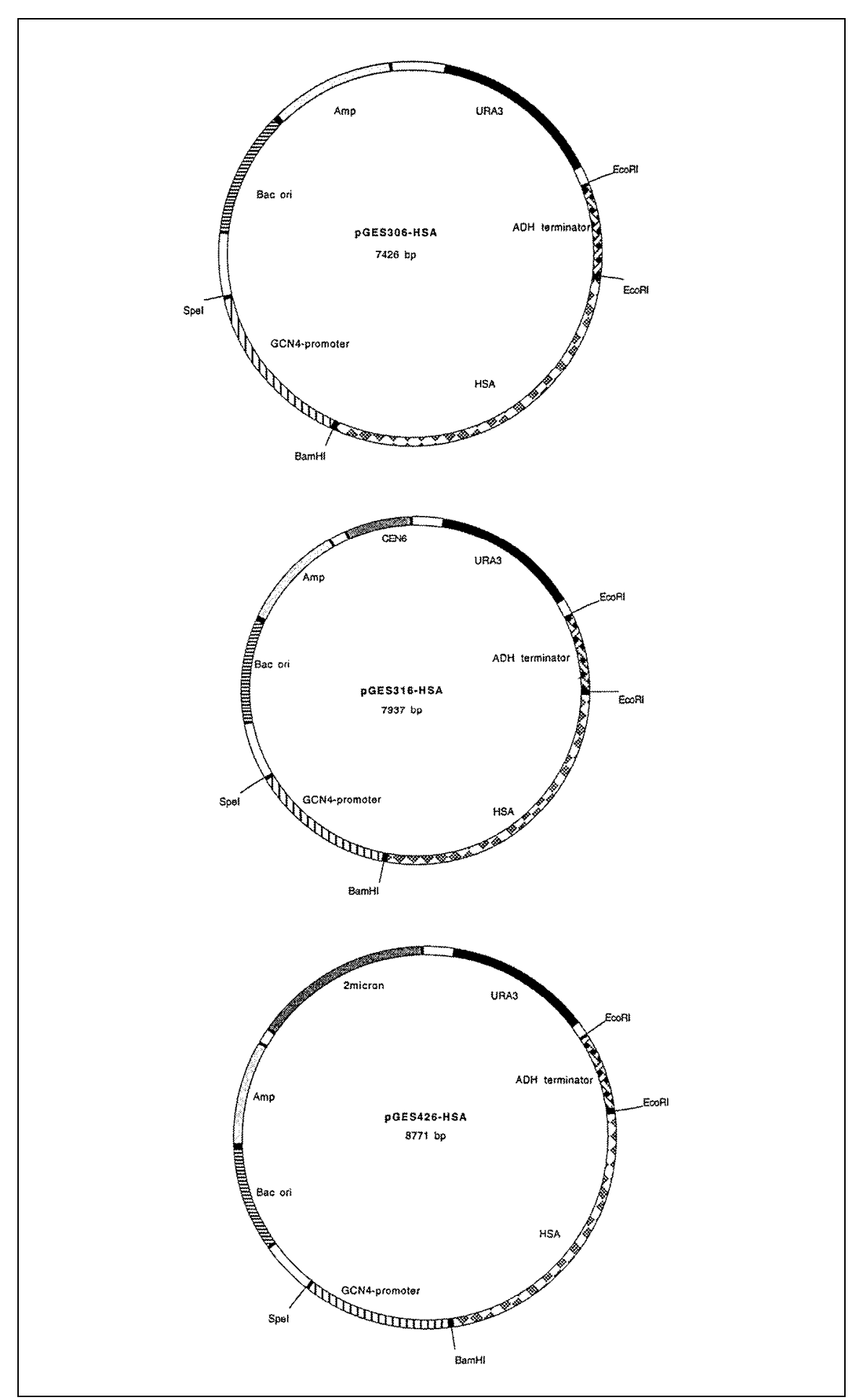

Figure 2. The basic pGES family containing the HSA cDNA. Note that pGES306-HSA is an integrative vector, pGES316-HSA is a centromeric vector and pGES426-HSA is a multicopy vector. All vectors shown contain the long GCN4 promoter (see text for details).
1:50 000. Peroxidase-conjugated goat anti-rabbit antibodies were purchased from Jackson ImmunoResearch Laboratories (West Grove, PA, USA) and used at the dilution of 1:25000. Puri- 
fied HSA (Sigma, St. Louis, MO, USA) was used as a standard positive control. RNA preparations and primer extension analysis were performed as previously described (6) using the following primers: HSA-extension (5'-ACCTCACTCTTGT-GTGCATCTC-3') and GCN4-extension (5'-ATAATTCGCTAGTGAAACTGATGGGC-3').

Primer extension products were separated on $6 \%$ acrylamide $7 \mathrm{M}$ urea gel.

\section{RESULTS AND DISCUSSION}

\section{A 1067 bp Fragment of the $G C N 4$ Upstream Sequences is Required for Efficient Expression of a Heterologous Gene}

To test whether regulatory elements of GCN4 could be used to regulate the expression of a heterologous gene, we cloned a DNA fragment that resides upstream from the GCN4 coding sequence. Initially, we cloned (from a genomic DNA prepared from the SP1 strain) a fragment (-706 to -1, designated short promoter; see Materials and Methods and Figure 1) previously shown to be sufficient for regulated expression of Gcn 4 and of a $G C N 4 / \beta$ galactosidase $(\beta$-gal) chimeric gene (containing 53 amino acids of Gcn4 fused to $\beta$-gal) $(13,14)$. The capability of this $706 \mathrm{bp}$ fragment alone (containing no GCN4 coding sequences) to control the transcription and translation

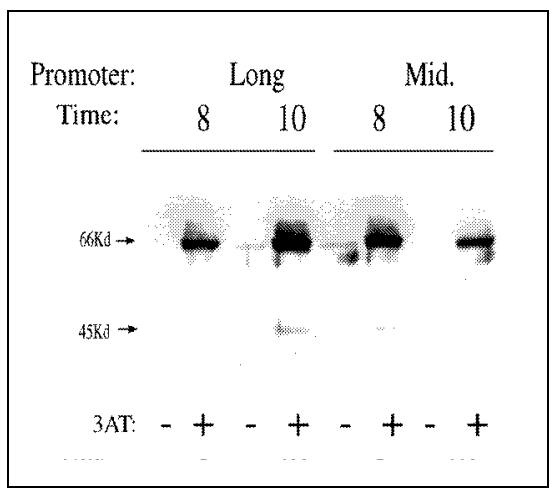

Figure 3. Induction and expression of HSA by pGES vectors containing either the long $G C N 4$ promoter (Long) or the middle-sized promoter (Mid.). Western blot analysis using anti-HSA antibodies was performed on protein extracts prepared from cultures (BJ2168 cells) harboring either pGES306-L-HSA (Long) or p306-M-HSA (Mid.) 8 and $10 \mathrm{~h}$ after addition of 3-AT. of another gene has not been reported. We constructed a GCN4-S-HSA (S stands for short promoter; see Materials and Methods) cassette that was introduced into a multicopy yeast plasmid as well as into centromeric and integrated plasmids. The terminator of the $A D H 1$ gene was inserted downstream to $G C N 4-\mathrm{S}-H S A$. The resulting plasmids were introduced into yeast cells that were subsequently grown under various conditions, including amino acid starvation and 3-AT treatment.

Protein extracts were prepared from the cultures, and the expression of HSA was monitored by Western blot analysis. Unexpectedly, in all cases, the expression of HSA was not detectable (data not shown). Therefore, we further cloned two longer fragments of GCN4 upstream sequences, one containing the sequence from -904 to -1 and another containing the sequence from -1067 to -1 (designated middle-sized and long promoters, respectively; see Figure 1 and Materials and Methods). When the $H S A$ gene was ligated to these fragments (plasmids pGES-M-HSA and pGES-L-HSA) and introduced into yeast cells, a high expression level of HSA was detected (Figure 3). Note that an additional band of $45 \mathrm{kDa}$ cross-reacted with anti-HSA antibodies. This band, which is a result of proteolytic activity of the Yap3 protease (19), was observed also by others that expressed HSA in yeast $(19,26)$.

In all constructs, HSA expression was barely detectable before induction with 3-AT, which suggests that expression from pGES plasmids is tightly controlled. Expression from constructs

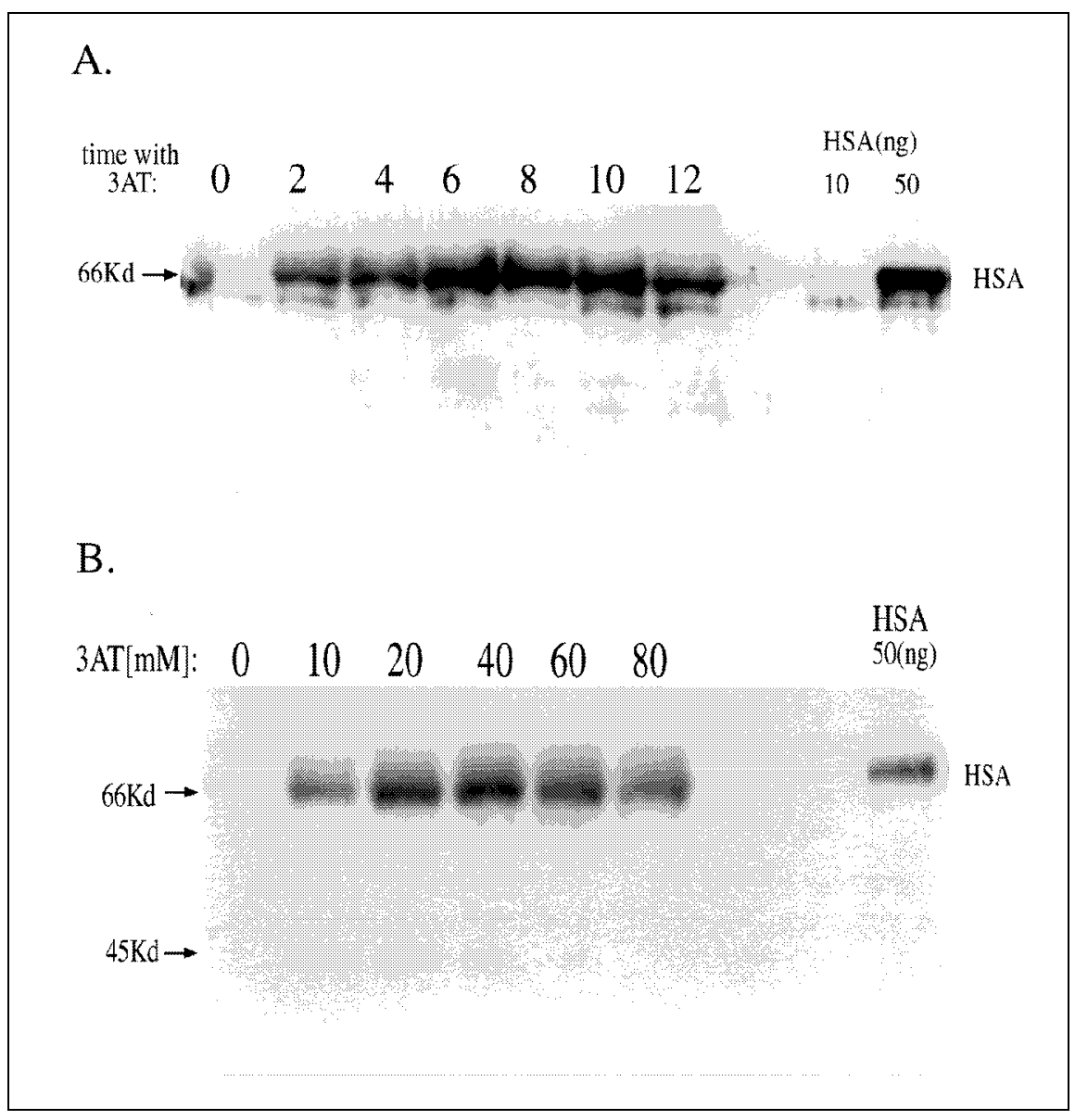

Figure 4. Time course and dose response analysis of induction of HSA expression. (A) A culture of BJ2168 cells harboring pGES426-L-HSA was grown to logarithmic phase and then supplemented with $20 \mathrm{mM}$ of 3-AT. Samples were removed at indicated time points and subjected to Western blot analysis. (B) A similar culture was grown to logarithmic phase and then divided into 6 fractions. Each fraction was supplemented with the indicated concentration of 3-AT. Cultures were further grown for $7 \mathrm{~h}$, collected and analyzed by Western blotting. Purified HSA was loaded on the right lanes of each gel. 
containing the long promoter was usually higher than that obtained with the middle-sized promoter (note, for example, the level of expression after $10 \mathrm{~h}$ induction; see Figure 3). All further experiments were carried out with the long promoter.

\section{Expression of HSA Under GCN4 Regulatory Elements Is Induced by 3-AT}

We tested whether the cloned GCN4 elements regulate HSA expression in a pattern similar to that of Gcn4 regulation. Yeast cells (strain BJ2168) that harbor pGES426-L-HSA were grown to logarithmic phase on $\mathrm{SD}$ (-uracil). Then, cells were collected and resuspended in SD(-uracil, -histidine) medium supplemented with $20 \mathrm{mM}$ 3-AT. The samples were removed from the culture at indicated time points, and the expression of HSA was measured. Two hours after the addition of 3-AT, the expression of HSA was easily detected. The expression reached its maximum level between 6 and $8 \mathrm{~h}$ after induction (Figure 4A). These results (Figure 3 and Figure $4 \mathrm{~A}$ ) show that a vector based on GCN4 upstream elements is useful for the inducible expression of foreign genes.

To test whether the level of expression could be finely controlled, we grew the BJ2168/pGES426-L-HSA cells to logarithmic phase, collected the cells, divided them into fractions and resuspended each fraction in medium supplemented with a different 3-AT concentration. The level of HSA expressed was proportional to the concentration of 3AT provided up to $40 \mathrm{mM}$. Higher concentrations of 3-AT reduced the expression level of HSA (Figure 4B).

\section{Expression of HSA from pGES Vectors Is Regulated at the Translational Level}

To verify that the induction of HSA expression occurred at the translational level, we measured the levels of $H S A$ mRNA and protein before and after induction with 3-AT (Figure 5). Protein extracts were tested by Western blot analysis using anti-HSA antibodies (Figure 5A), while RNA preparations were subjected to primer extension analysis. The extended primers were separated on sequencing gels, which allowed accurate detection of each mRNA initiation site (Figure 5, B and C). As determined by Hinnebusch (14), the transcription of GCN4 initiates at three major sites $(+1,+18$ and +30$)$ and at several minor sites, which gives rise to several mRNA species. Figure 5C shows that, as in the case of GCN4 mRNA, the HSA mRNA is expressed at a similar level before and after 3-AT is provided. Most initiation sites used by RNA polymerase to transcribe the GCN4/HSA mRNA are identical to those found in GCN4 mRNAs (compare Figure 5, B and C). Curiously, the level of GCN4 mRNA in cells that ex-

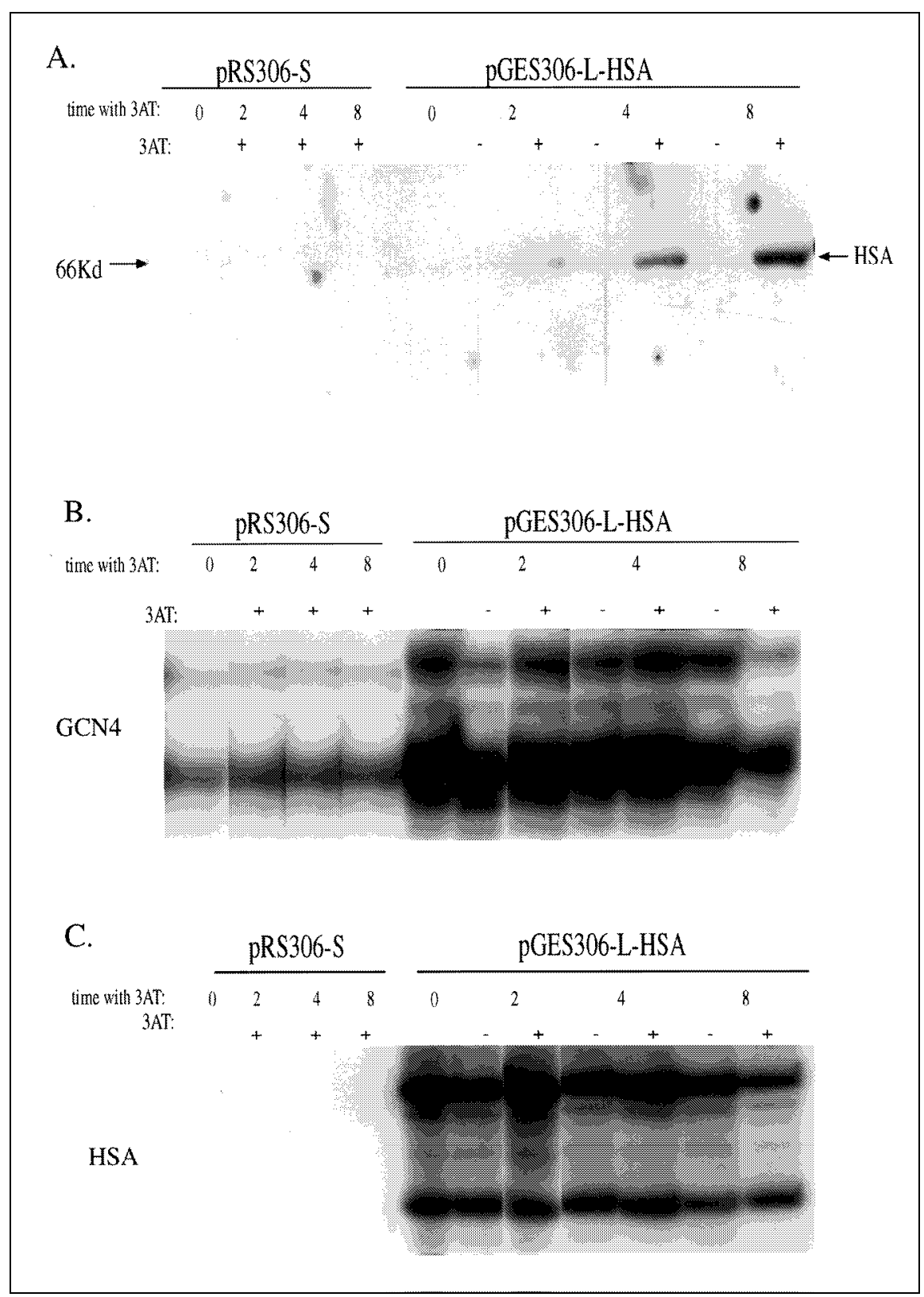

Figure 5. Expression of HSA from pGES vectors is induced at the translational level. A culture harboring an integrated copy of pGES306-L-HSA and a culture harboring an integrated copy of the control plasmid pRS306-S (a similar vector but with no HSA cDNA) were grown to logarithmic phase when 3AT was added. At the indicated time points, the samples were removed. Protein extracts and RNAs were prepared from each sample and analyzed by Western blot and primer extension analysis, respectively. (A) Western blot analysis. (B) Primer extension analysis using GCN4 primer. (C) Primer extension analysis using $H S A$ primer. 
press HSA is high (Figure 4B). The reason for this phenomenon is unknown to us. In contrast to the constitutive and uniform expression of the HSA mRNA, the HSA protein was barely detectable before induction with 3-AT, and its expression increased in time following induction. We conclude that 3-AT-dependent induction of HSA expression occurs at the translational level.

\section{GCN4-Based Vector Induces Efficient Expression as an Integrated Copy}

The transcription rate of $G C N 4$ seems to be high under most growth conditions in various strains and mutants $(5,14)$. Accordingly, mRNA levels of $H S A$ expressed from pGES plasmids do not change significantly under different growth conditions (Figure 5 and data not shown). We assume therefore that the efficient expression from the pGES426-L-HSA stems mainly from the efficient translation rate and less from the high copy number of the plasmid. If this is the case, we should be able to use the GCN4/HSA cassettes as integrated copies. Cells carrying an integrated vector could be grown on YPD medium to high cell densities and produce high yields of HSA.

To test this possibility, we have inserted the GCN4-L-HSA fragment into an integrative vector and thereby obtained the pGES306-L-HSA plasmid (see Materials and Methods). This construct was integrated into the yeast genome, and the resulting clones were grown on rich nonselective media to a high cell concentration $\left(5 \times 10^{8}\right.$ cells $/ \mathrm{mL}$ ) before the addition of 3-AT. Under these conditions, the expression of HSA was still efficiently induced (Figure 6). The level of HSA obtained per cell was just slightly less than that obtained using the multicopy pGES426-L-HSA vector. However, as the culture grown on YPD reached higher cell concentration, the quantity of intracellular HSA protein obtained (about $0.5 \mathrm{mg} / \mathrm{L}$ ) was about two times higher of that obtained with the multicopy plasmid pGES426-L-HSA. Notably, all experiments described in this study were performed on cultures grown in laboratory flasks, conditions that do not support maximal yields.

To compare the pGES vector to commonly used vectors, we have ligated the cDNA that encodes HSA downstream from the $A D H 1$ promoter in the vector $\mathrm{pAD} 4 \Delta(1)$. Cultures that harbor either pADH-HSA or pGES426-LHSA were grown in parallel, and the expression of HSA was compared. In the case of pGES426-L-HSA, expression was compared after induction. The levels of HSA expression obtained from both plasmids were similar. Using commercial purified HSA as a standard, we calculated that about $250 \mu \mathrm{g} / \mathrm{L}$ of intracellular HSA were obtained in this experiment, about half of the amount obtained using the integrative version (pGES306-L-HSA) on rich medium. We conclude that the pGES system could be more efficient than currently available yeast expression vectors.

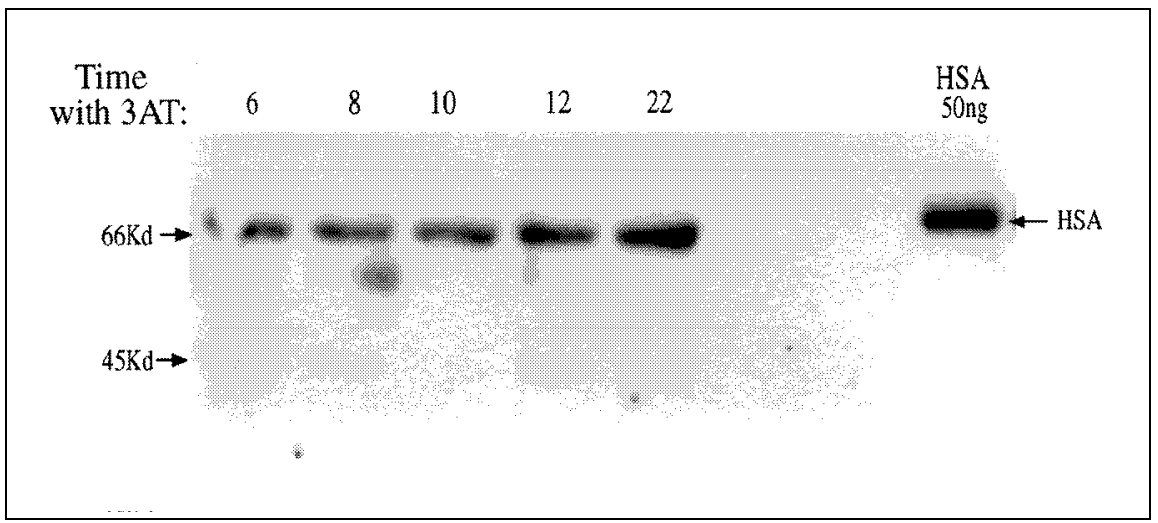

Figure 6. pGES system is inducible in highly concentrated cultures. A culture harboring an integrated copy of pGES306-L-HSA was grown on YPD medium to $\mathrm{A}_{600}=3.3$ when the medium was replaced with SD(-histidine) supplemented with $40 \mathrm{mM} 3$-AT. Samples were removed and analyzed by Western blot analysis at the indicated time points after induction. Purified HSA was loaded on the right lane of the gel. 
Although pGES plasmids already seem quite efficient, they could be regarded as archetypes that could be further manipulated and improved. For example, it might be possible to increase the mRNA level of HSA through a combination of a strong constitutive promoter (of another gene) with the $5^{\prime}$ UTR of GCN4. Such a chimera might be more efficient than pGES and still be translationally regulated. The regulation of GCN4 translation is well studied, and mutants are available in which the GCN4 translation rate is constitutively high and not regulated ( $g c d$ mutants) $(11,14,17)$. Also, GCN4 translation is induced by the yeast Ras/cAMP cascade and is high in $R A S^{\text {vall9 }}$ and $b c y l$ strains (5). The pGES plasmids could be used in $g c d$ strains and in RAS2vall9 and $b c y l$ strains as constitutive vectors. These ideas are only a few of the many ways in which the pGES vectors could be used to obtain large quantities of proteins for research and industry.

\section{ACKNOWLEDGMENTS}

We wish to thank Prof. Sergei Braun for critically reading the manuscript.

\section{REFERENCES}

1.Ballester, R., T. Michaeli, K. Ferguson, H.P. Xu, F. McCormick and M. Wigler. 1989. Genetic analysis of mammalian GAP expressed in yeast. Cell 59:681-686.

2.Chritianson, T.W., R.S. Sikorski, M. Dante, J.H. Shero and P. Hieter. 1992. Multifunctional yeast high-copy-number shuttle vectors. Gene 110:119-122.

3.De Baetselier-Van Broekhoven, A. 1994. Yeast as source for therapeutic and diagnostic proteins, p. 431-447. In Y. Murooka and T. Imanaka (Eds.), Recombinant Microbes For Industrial and Agricultural Applications. Marcel Dekker, New York.

4.Dever, T.E., L. Feng, R.C. Wek, A.M. Cigan, T.F. Donahue and A.G. Hinnebusch. 1992. Phosphorylation of initiation factor $2 \alpha$ by protein kinase GCN2 mediates gene-specific translational control of GCN4 in yeast. Cell 68:585-596.

5.Engelberg, D., C. Klein, H. Martinetto, K. Struhl and M. Karin. 1994. The UV response involving the Ras signaling pathway and AP-1 transcription factors is conserved between yeast and mammals. Cell 77:381390.

6.Engelberg, D., E. Zandi, C.S. Parker and M. Karin. 1994. The yeast and mammalian Ras pathways control transcription of heat shock genes independently of heat shock tran- scription factor. Mol. Cell. Biol. 14:49294937.

7.Gao, B., S.C. Flores, S.K. Bose and J.M. McCord. 1996. A novel Escherichia coli vector for oxygen-inducible high-level expression of foreign genes. Gene 176:269-272.

8.Garreau, H., J.H. Camonis, C. Guitton and M. Jacquet. 1990. The Saccharomyces cerevisiae CDC25 gene product is a $180 \mathrm{kDa}$ polypeptide and is associated with a membrane fraction. FEBS Lett. 269:53-59.

9.Gold, L. 1990. Expression of heterologous proteins in Escherichia coli. Methods Enzymol. 185:11-14.

10.Harashima, S. 1994. Heterologous protein production by yeast host-vector systems, $\mathrm{p}$. 137-158. In Y. Murooka and T. Imanaka (Eds.), Recombinant Microbes for Industrial and Agricultural Applications. Marcel Dekker, New York.

11.Harashima, S. and A. Hinnebusch. 1986. Multiple GCD genes required for repression of GCN4, a transcriptional activator of amino acid biosynthetic genes in Saccharomyces cerevisiae. Mol. Cell. Biol. 6:3990-3998.

12.Hill, D.E., I.A. Hope, J.P. Macke and K. Struhl. 1986. Saturation mutagenesis of the yeast his3 regulatory site: requirements for transcriptional induction and for binding by GCN4 activator protein. Science 234:451457.

13.Hinnebusch, A.G. 1984. Evidence for translational regulation of the activator of general amino acid control in yeast. Proc. Natl. Acad. Sci. USA 81:6442-6446

14.Hinnebusch, A.G. 1985. A hierarchy of trans-acting factors modulates translation of an activator of amino acid biosynthetic genes in Saccharomyces cerevisiae. Mol. Cell. Biol. 5:2349-2360.

15.Hinnebusch, A.G. 1988. Mechanisms of gene regulation in the general control of amino acid biosynthesis in Saccharomyces cerevisiae. Microbiol. Rev. 52:248-273.

16.Hinnebusch, A.G. 1992. General and pathway-specific regulatory mechanisms controlling the synthesis of amino acid biosynthetic enzymes in Saccharomyces cerevisiae, p. 319414. In J.R. Broach, E.W. Jones and J.R. Pringle (Eds.), The Molecular and Cellular Biology of the Yeast Saccharomyces. Vol. 2: Gene Expression. CSH Laboratory Press, Cold Spring Harbor, NY.

17.Hinnebusch, A.G. 1997. Translational regulation of yeast GCN4. J. Biol. Chem. 272:21661-21664.

18.Kataoka, T., S. Powers, C. McGill, O. Fasano, J. Strathern, J. Broach and M. Wigler. 1984. Genetic analysis of yeast RAS1 and RAS2 genes. Cell 37:437-445.

19.Kerry-Williams, S.M., S.C. Gilbert, L.R. Evans and D.J. Ballance. 1998. Disruption of the Saccharomyces cerevisiae YAP3 gene reduces the proteolytic degradation of secreted recombinant human albumin. Yeast 14:161-169.

20.Mattanovich, D., R. Weik, S. Thim, W. Kramer, K. Bayer and H. Katinger. 1996. Optimization of recombinant gene expression in Escherichia coli. Ann. N.Y. Acad. Sci. USA 782:182-190.

21.Romanos, M.A., C.A. Scorer and J.J. Clare.
1992. Foreign gene expression in yeast: a review. Yeast 8:423-488.

22.Rose, A.B. and J.R. Broach. 1990. Propagation and expression of cloned genes in yeast: $2-\mu \mathrm{m}$ circle-based vectors. Meth. Enzymol. 185:234-279.

23.Schena, M., D. Picard and K.R. Yamamoto. 1991. Vectors for constitutive and inducible gene expression in yeast. Meth. Enzymol. 194:389-398

24.Schneider, J.C. and L. Guarente. 1991. Vectors for expression of cloned genes in yeast: regulation, overproduction, and underproduction. Meth. Enzymol. 194:373-388.

25.Sikorski, R.S. and P. Hieter. 1989. A system of shuttle vectors and yeast host strains designed for efficient manipulation of DNA in Saccharomyces cerevisiae. Genetics 122:1927

26.Sleep, D., G.P. Belfield and A.R. Goodey. 1990. The secretion of human serum albumin from the yeast Saccharomyces cerevisiae using five different leader sequence. Biotechnology 8:42-46.

27.Walsh, G. and D.R. Headon. 1994. Protein Biotechnology. John Wiley \& Sons, West Sussex, England.

28. Yasueda, H. and H. Matsui. 1994. Overproduction of heterologous proteins in Escherichia coli, p. 49-70. In Y. Murooka and T. Imanaka (Eds.), Recombinant Microbes For Industrial and Agricultural Applications. Marcel Dekker, New York.

29.Zubenko, G.S., A.P. Mitchell and E.W Jones. 1980. Mapping of the proteinase B structural gene PRB1, in Saccharomyces cerevisiae and identification of nonsense alleles within the locus. Genetics 96:137-146.

Received 7 April 1999; accepted 22 November 1999.

\section{Address correspondence to:}

Dr. David Engelberg

Dept. of Biological Chemistry

The Institute of Life Sciences

The Hebrew University of Jerusalem

Jerusalem 91904, Israel

Internet: engelber@vms.huji.ac.il 